\title{
Educación Secundaria de Jóvenes y Adultos. Reseña de un libro urgente
}

Reseña del libro Educación Secundaria de Jóvenes y adultos. Políticas, discursos y modos de gestión en torno a la inclusión educativa A. Homar y G. Altamirano (Comps.) (2018). Buenos Aires: Noveduc

\section{( Juan Pablo Castañeda Agüero} Universidad de Buenos Aires, Facultad de Filosofía y Letras, Argentina

El libro titulado Educación Secundaria de Jóvenes y adultos. Políticas, discursos y modos de gestión en torno a la inclusión educativa, publicado por Noveduc en septiembre de 2018, tiene como autoras/es de sus capítulos a un equipo de investigadoras/es de la FHAyCS-UADER con trayectoria en investigación y docencia dentro del campo de la Educación de Jóvenes y Adultos: Amalia Homar (Dir.), Gisela Altamirano, Paula Dalinger, Alfonsina Francisconi, Ma. del Carmen Ulrich, Mario A. Villanueva y Ma. Eugenia Bordón. El libro es producto de un proceso de investigación originado en el marco de la convocatoria realizada en 2013 por el Programa Nacional "Hacia un Consenso del Sur para el Desarrollo con Inclusión Social", de la Secretaría de Políticas Universitarias (Ministerio de Educación de la Nación); donde han participado profesoras y estudiantes de la cátedra de Investigación Educativa de los profesorados de Educación Secundaria de la FHAyCS-UADER.

La trayectoria de este equipo de investigadoras/es otorga consistencia al planteo de sus preguntas, tratándose de preocupaciones claves que dan cuenta de la complejidad del campo, sus avances y deudas en el último periodo en materia de inclusión educativa. En palabras de las autoras,

buscamos desentrañar las relaciones que se construyen entre políticas estatales, ofertas y trayectorias educativas, que sostienen la inclusión de jóvenes y adultos desde los marcos nacionales y provinciales, las prácticas institucionales y los modos de gestión de quienes tienen la responsabilidad política de ejecutarlas desde el gobierno de la educación de cada provincia.

Para ello se ubican en el lapso 2004-2015, dando cuenta del contexto social, educativo, político y legislativo que implicó este periodo, centrado en el plano educativo por la preocupación en torno a la inclusión socioeducativa plasmada fundamentalmente en la Ley de Educación Nacional (LEN) del año 2006. En este sentido, las propias autoras advierten que la obligatoriedad definida por la LEN viene a poner en cuestión el carácter históricamente selectivo del nivel y su función social. Con esta consideración, a lo largo del libro se establece un diálogo permanente con el periodo anterior caracterizado por las políticas neoliberales en lo económico, neoconservadoras en lo político y signado en el plano educativo por la Ley Federal de Educación.

El estudio se ubica en las provincias argentinas de Entre Ríos, Chaco y Córdoba, marcando una clara intencionalidad de un análisis comparativo. Esta decisión es fundamentada tanto por la necesidad de poner el foco por fuera de la provincia de Buenos Aires, donde se ubican el mayor número de investigaciones; como también por la relevancia de considerar las diferencias educativas, contextuales y sociales entre cada una de dichas provincias. Esta decisión se traduce en un enriquecimiento del análisis, permitiendo visibilizar contradicciones en el entramado legislativo y su recreación institucional, como así también desigualdades sociales y educativas que aún persisten.

El libro, estructurado en cinco capítulos, aborda la problemática de la inclusión en la educación secundaria de jóvenes y adultos (ESDJA) desde distintas dimensiones, combinando perspectivas teórico-metodológicas para su abordaje.

En el primer capítulo, titulado "Educación secundaria de jóvenes y adultos. Perspectivas y debate", las autoras dan cuenta del marco teórico desde el cual comprenden y trabajan el objeto de estudio. Parten de una concepción sobre la Educación Permanente que no se restringe a la nominación empleada por la LEN para la modalidad orientada a Jóvenes y Adultos, sino que implica una serie de supuestos políticos pedagógicos para entender y demandar el derecho por la educación a lo largo de toda la vida. A su vez, se ubica el objeto de estudio en su perspectiva histórica y como campo de disputa de sentidos, relaciones sociales y políticas, recuperando diversos conceptos y perspectivas teóricas provenientes de la teoría social crítica. Se recupera a su vez, de la 
mano con la categoría de inclusión, la perspectiva de las trayectorias educativas para dar a conocer las contradicciones devenidas a partir de la expansión de la escuela media.

Recuperando el concepto de María Teresa Sirvent de situación educativa de riesgo, las autoras analizan el Censo Nacional de Población, Hogares y ViviendasINDEC del año 2010, para dar cuenta de la situación educativa de la población de 15 años y más que asistió y ya no asiste a la escuela. De este modo, vuelven a verificar altos porcentajes de población de 15 años y más que se encuentra en situación educativa de riesgo, es decir, que llegó hasta la primaria incompleta, completa o a lo sumo hasta la secundaria incompleta como máximo nivel educativo alcanzado; encontrándose con una probabilidad estadística de quedar marginado de la vida social, política y económica. No obstante, las autoras identifican un crecimiento sostenido (aunque desigual según jurisdicción) de la matrícula de la modalidad en el periodo 2004-2015, con un claro predominio de la educación secundaria. Aun así, la demanda efectiva sigue representando en todos los casos un porcentaje mínimo (alrededor del $5 \%$ ) de la demanda potencial por educación de jóvenes y adultos tanto a nivel país como en las jurisdicciones estudiadas. En este sentido -concluyen las autoras-, estos datos dan cuenta de la necesidad de políticas educativas para la modalidad a fin de alcanzar los derechos establecidos por la LEN. Por otra parte, advierten la complejidad de las dinámicas de inclusión/exclusión educativa, no solo referidas al ingreso o expulsión del sistema escolar sino también con relación a las dinámicas de exclusión en torno al conocimiento ocurridas al interior de las instituciones escolares.

Finalmente, este primer capítulo propone un interesante, exhaustivo y actualizado estado de situación en torno a la producción científica en el campo de la Educación de Jóvenes y adultos en Argentina, identificando distintos núcleos problemáticos para su organización: políticas y prácticas de inclusión socioeducativa en la educación de jóvenes y adultos; las trayectorias socioeducativas y las biografías escolares de los jóvenes y adultos; $y$ prácticas institucionales, sujetos y contextos. En las reflexiones finales del capítulo, evidencian una realidad en torno al campo que es compartida a su vez por otros/as investigadores/as: el carácter marginal de esta modalidad dentro del campo de la investigación educativa, más aún al tratarse de estudios anclados en las provincias del país. De aquí también el valor de esta producción científica, que plantea el desafío de

impulsar investigaciones que realicen aportes para repensar el diseño de políticas educativas, estrategias de acompañamiento a las trayectorias socioeducativas de jóvenes y adultos, los dispositivos institucionales de inclusión, la formación y prácticas de los docentes de la modalidad, el sentido de la educación permanente y popular, y la articulación con la formación laboral.

El segundo capítulo, titulado "El enfoque metodológico en la construcción de resultados", expone la perspectiva metodológica a partir de la cual las autoras abordaron su objeto de estudio. Se plantea la definición de dos objetivos que guiaron las consecuentes decisiones metodológicas: uno, "analizar la inclusión social y educativa en la educación secundaria de jóvenes y adultos en el contexto de las políticas de Estado en Entre Ríos, Chaco y Córdoba (Argentina) en el periodo 2004-2015·; y dos,

interpretar las relaciones que se construyen entre políticas estatales, ofertas institucionales y trayectorias educativas que sostienen la inclusión de jóvenes y adultos desde los marcos normativos y discursos de gestión de quiénes tienen la responsabilidad de conducir la educación permanente de jóvenes y adultos en las provincias seleccionadas.

Aquí se destaca la decisión de asumir una convergencia de lógicas que combinan estrategias cualitativas y cuantitativas con el propósito de producir una triangulación de fuentes de información. Desde la perspectiva cualitativa -señalan las autoras-, se pretende indagar las normativas, documentos oficiales y entrevistas en profundidad a funcionarios responsables de la gestión de la ESDJA. En cuanto a la lógica cuantitativa, ha sido trabajada para el estudio de las ofertas educativas, la población destinataria de la modalidad y el estudio de su demanda potencial y efectiva en las provincias seleccionadas. Al mismo tiempo, se describen las actividades realizadas por el equipo combinando los procesos de indagación, análisis y construcción del marco teórico y categorías de análisis. En tal sentido, este capítulo resulta de especial interés para pensar la investigación educativa en este campo asumiendo su complejidad; abordando la investigación como un proceso no lineal y permanentemente sometido a la reflexión para la toma de decisiones. Dando cuenta de este proceso, las autoras también señalan las dificultades y desafíos que fueron asumiendo con relación al acceso a determinadas fuentes, su selección y tratamiento a través de diversos instrumentos.

El siguiente capítulo, "Entre las políticas educativas y los dilemas de la gestión”, parte del reconocimiento 
de que durante el lapso 2004-2015, desde la sanción de la LEN, se desarrolló un marco normativo nacional y provincial que tendió al diseño e implementación de planes y programas orientados a mejorar las condiciones materiales y simbólicas de sujetos e instituciones destinatarios de la Modalidad. En este sentido - señalan-, el Estado vuelve a reasumir (a diferencia del periodo anterior) sus obligaciones con el campo educativo en general y la ESDJA en particular. Para el abordaje de este análisis, las autoras asumen una mirada crítica acerca de la creación y recreación de las políticas públicas, poniendo especial interés en las articulaciones entre los procesos micro y macropolíticos, y en la acción de los distintos actores sociales intervinientes. De este modo, se trabaja en torno a dos nudos centrales para el análisis de las políticas educativas: a) textos legales, documentos oficiales, resoluciones del Consejo Federal de Educación y normativas de las tres provincias estudiadas; b) tensiones y rupturas en la gestión de las políticas educativas para la educación secundaria de jóvenes y adultos; y recontextualización de las políticas educativas nacionales. En lo que atañe al primer nudo, referido a los textos normativos, las autoras se preguntan acerca del "porqué de su formulación, los espacios de discusión que habilitan, quién/ es son los destinatarios, los intersticios y/o zonas grises, cuáles son las continuidades y rupturas que se plantean respecto a las normativas que reemplazan, entre otros aspectos". Con relación al segundo nudo, analizan "las connotaciones que adquieren las políticas educativas en los discursos de los referentes políticos entrevistados". A lo largo del tercer capítulo, estos nudos se van trabajando, a su vez, en clave de rupturas y continuidades con relación al período anterior a 2004. Se identifican contradicciones y avances tanto relacionados con la definición político-pedagógica de la Modalidad, por ejemplo respecto a la persistencia de su carácter compensatorio, como en su recreación por parte de los actores centrales de la gestión educativa en cada jurisdicción. En este sentido, también se identifican diferencias jurisdiccionales importantes. Los debates señalados en este capítulo cobran especial relevancia y vigencia en el escenario político actual, donde el derecho a la educación permanente se ha vuelto a poner en entredicho, amenazado tanto por un contexto de agudización de las múltiples pobrezas, como por las orientación de políticas educativas que han limitado la tendencia a una mayor inclusión de las personas jóvenes y adultas al sistema educativo.

El cuarto capítulo se titula "Organización institucional y definiciones curriculares. Diversidad y fragmentación". En él, las autoras proponen pensar las instituciones educativas de la ESDJA como campos en disputa. Las tensiones entre continuidad y ruptura se vuelven a manifestar en su dimensión institucional, y allí ponen el foco de análisis en dos ejes: 1) la organización institucional que evidencia la fragmentación de las propuestas formativas; y 2) las particularidades jurisdiccionales en las definiciones curriculares. En el desarrollo del primer eje, las autoras advierten un claro posicionamiento por parte de la normativa del periodo estudiado, en pensar los centros educativos de la modalidad de manera diferenciada al formato escolar tradicional. Al analizar las provincias seleccionadas, encuentran una gran diversidad institucional; destacándose en Córdoba la mayor coherencia con respecto a las definiciones nacionales. En Chaco se advierte una clara fragmentación organizativa y pedagógica, a la vez que se identifica una falta de reconocimiento a la especificidad de las propuestas de ESDJA. Y en Entre Ríos, por su parte, se analiza la doble dependencia de las instituciones de la modalidad, compartida con la Dirección de Educación Secundaria. Con relación al eje 2), referido a las definiciones curriculares, las autoras concluyen que "la existencia de lineamientos federales sobre las características de las instituciones y la estructura curricular de la modalidad no garantiza su plena ejecución ni se traduce de manera idéntica en las prácticas en las jurisdicciones estudiadas". En este sentido, se logra un rico análisis comparativo entre las tres provincias, el cual da cuenta de la recreación particular de los lineamientos federales en cada jurisdicción.

Finalmente, el quinto capítulo, "La inclusión socioeducativa de los sujetos y sus trayectorias", vuelve a explicitar la combinación de lógicas de indagación por parte de los/as autores/as, trabajando tanto con datos censales para visibilizar las trayectorias educativas de jóvenes y adultos, recuperando los conceptos de demanda potencial y demanda efectiva, como también mediante el análisis de normativas y entrevistas realizadas a actores claves de la gestión educativa de esta modalidad. Analizando las políticas de Estado desplegadas en el periodo 2004-2015, se destaca la consideración de la EDJA como permanente, renovando la mirada sobre los sujetos pedagógicos y la concepción de la modalidad misma. Si bien se logra identificar en este análisis una "adecuación" del discurso de los funcionarios al cambio de perspectiva propuesto por la LEN y las posteriores normativas, los/ as autores/as marcan importantes aspectos que tensionan la posibilidad de pensar un sentido único de la política educativa; por ejemplo en relación con cómo es pensado el sujeto destinatario de la modalidad. Se advierte en la normativa, a su vez, una marcada impronta que focaliza la trayectoria educativa en la dimensión escolar, tensionando el concepto mismo de educación permanente. Por último, destaca la creación 
de programas orientados a mejorar las condiciones materiales y simbólicas de las personas e instituciones de la modalidad. No obstante, el análisis de los datos estadísticos continuaría mostrando que existe un profundo desfasaje entre la demanda efectiva y la demanda potencial por ESDJA, al poner de relieve "la gravedad del estado de situación en la que se encuentra esta población respecto de la obligatoriedad enunciada en las normativas". En esta dirección -concluyen-, si bien hubo un crecimiento en el ingreso de estudiantes a la modalidad durante el lapso analizado, aún continúan fuera del sistema educativo gran parte de la población destinataria de la ESDJA.
Como ya ha sido señalado, este libro logra presentar múltiples aportes teóricos y metodológicos al campo del conocimiento de la EDJA, abordando críticamente una multiplicidad de dimensiones que dan cuenta de la complejidad del campo: actualiza debates referidos a la política educativa, denuncia las desigualdades educativas y las deudas referidas a una efectiva universalización del derecho a la educación permanente, y plantea nuevas preguntas que interpelan el periodo actual. De aquí su urgencia y necesaria lectura. 